\title{
OPEN Oral administration of resveratrol or lactic acid bacterium improves lens elasticity
}

\author{
Hayato Nagashima ${ }^{1,2}$, Nobunari Sasaki ${ }^{1,3}$, Sachie Amano ${ }^{1}$, Shigeru Nakamura ${ }^{1}$, \\ Motoshi Hayano ${ }^{1,3}$ \& Kazuo Tsubota ${ }^{1,3}$
}

A decrease in the elasticity of the ocular lens during aging is associated with loss of the accommodative ability of the eye, leading to presbyopia. Although near vision impairment is a social issue affecting the length of healthy life expectancy and productivity of elderly people, an effective treatment to improve near vision has not yet become available. Here we examined the effect of Enterococcus faecium WB2000, Lactobacillus pentosus TJ515, and resveratrol on lens elasticity in rats, where the stiffness of the ocular lens increases exponentially during the aging process. A combination of WB2000 and resveratrol improved lens elasticity not only in the long term but also with just shortterm treatment. In addition, TJ515 decreased stiffness in the eye lens with long-term treatment. Therefore, the oral administration of WB2000 and resveratrol or TJ515 may be a potential approach for managing the progression of near vision impairment.

The accommodation ability to focus on near subjects is known to be decreased with age. The near vision impairment and presbyopia affect 1.8 billion people in 2015 (25\% of people worldwide), with the number expects to reach 2.1 billion in 2030 given the expanding global population ${ }^{1}$. Although the near vision impairment has an impact on a healthy life expectancy and work productivity, current treatments remain limited to wearing glasses or contact lenses and surgical intraocular lens insertion ${ }^{2}$.

Lens elasticity is known to correlate with the near vision impairment in humans and the stiffness of the lens in the cortex and nuclear region is drastically increased with aging ${ }^{3-6}$. Pirenoxine eye drops prevented hardening of the lens in rats and Pirenoxine improved the accommodation ability in human in a clinical study ${ }^{7}$. Anti-oxidant supplementation and periocular warming were also observed to improve accommodation in human ${ }^{8,9}$. Lens fiber cells in the cortex and nuclear region are differentiated from lens epithelial cells accompanied by the loss of subcellular organelles including nuclear DNA in an autophagy-independent manner ${ }^{10}$. Given that protein synthesis and turnover are completely abolished in lens fiber cells, the maintenance of long-lived protein is crucial for continued lens homeostasis. Posttranslational modifications, such as disulfide bonding, deamidation, glycation, and oxidation, are increased with age, with the greater rate of disulfide bond formation in $\alpha-, \beta-$, and $\gamma$-crystallin capable of inducing the appearance of high-molecular-weight crystalline ${ }^{5,11-16}$. Existing research suggests the administration of lipoic acid choline ester (LACE), derived from lipoic acid, reduced the concentration of disulfides in protein in mice, leading to the restoration of lens elasticity ${ }^{17}$. Some droplets including LACE are currently undergoing clinical trials to evaluate their efficacy and safety as the development of a new therapeutic agent to restore accommodative amplitude is of great interest ${ }^{18}$.

Resveratrol, a polyphenol found in grape or other plants, has shown an antioxidant capacity in neurodegenerative disease, the aging heart, and vascular diseases as well as the ability to facilitate lifespan elongation ${ }^{19}$. Nonspecific thiol oxidation induces disulfide binding, leading to protein aggregation. Antioxidative activity may play a crucial role in mitigating age-related ocular diseases such as glaucoma and age-related macular disease through the inhibition of reactive oxygen $(\mathrm{ROS})^{20-25}$. However, though resveratrol is known to prevent oxidative stress in human lens epithelial cells, its effects on lens stiffness and the progression of the near vision impairment remain unknown.

Moreover, lactic acid bacteria, one of the groups of probiotic bacteria, have biological functions including antioxidative properties ${ }^{26-29}$. The long-term administration of a lactic acid bacterium suppressed retinal inflammation and retinal cell loss in aged mice ${ }^{30}$.

\footnotetext{
${ }^{1}$ Department of Ophthalmology, Keio University School of Medicine, 35 Shinanomachi, Shinjuku-ku, Tokyo 160-8582, Japan. 'Department of Plastic and Reconstructive Surgery, Keio University School of Medicine, Tokyo, Japan. ${ }^{3}$ Tsubota Laboratory, Inc., Tokyo, Japan. ${ }^{\circledR}$ email: tsubota@z3.keio.jp
} 

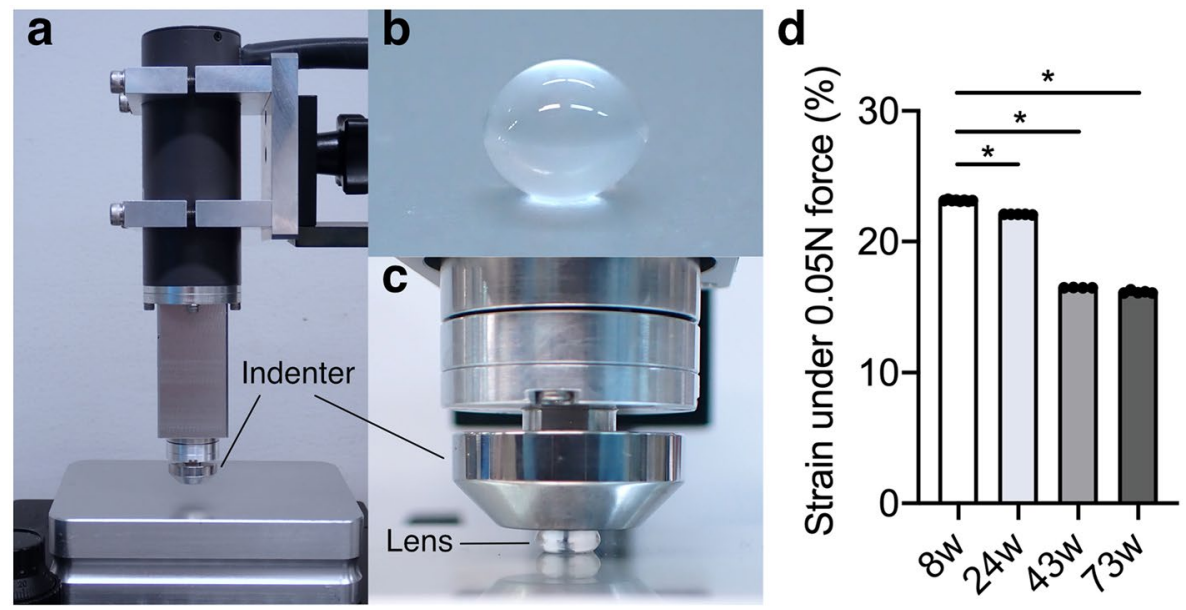

Figure 1. The measuring instrument, rat lens sample and the elasticity of young, middle and aged rat lenses. (a) The force-displacement measuring instrument with flat indentation. (b,c) The rat lens sample were put on a stage and a $12 \mathrm{~mm}$-diameter flat indenter applied a load on the lens along the anteroposterior axis of the lens from above to measure the force and indentation displacement until $5.0 \mathrm{~N}(\mathrm{~N})$ with the speed of $0.1 \mathrm{~mm} / \mathrm{s}$. (d) The lenses extracted from young (8-week-old, $\mathrm{n}=8$ ), middle-aged (24-week-old and 43-week-old) and old-aged (73-week-old, $n=5$ ) male rats were assessed. The percentages of strain for the four age groups when lenses were pushed with 0.05 newtons $(\mathrm{N})$ of force are shown in the bar graph. Data are presented as mean \pm SEM. The asterisk indicates a statistically significant difference compared to 8 -week-old $\left({ }^{\star} P<0.05\right.$, one-way analysis of variance followed by Tukey's post-hoc test).

\begin{tabular}{|l|l|l|l|l|}
\hline Age & Strain (\%) & \% of strain (compared to 8-week-old) & Sample number & $\boldsymbol{P}$ value (compared to 8-week-old) \\
\hline 8-week-old & $23.14 \pm 0.04$ & 100 & 8 & - \\
\hline 24-week-old & $22.08 \pm 0.02$ & 98.5 & 5 & $<0.0001^{*}$ \\
\hline 43-week-old & $16.47 \pm 0.01$ & 71.2 & 4 & $<0.0001^{*}$ \\
\hline 73-week-old & $16.14 \pm 0.10$ & 69.7 & 5 & $<0.0001^{*}$ \\
\hline
\end{tabular}

Table 1. The elasticity of young, middle and aged rat lenses was measured with a force-displacement measuring instrument. As shown in Fig. 1d, the lenses extracted from young (8-week-old, $n=8$ ), middle (24-week-old, $\mathrm{n}=5$ and 43-week-old, $\mathrm{n}=4$ ) and aged (73-week-old, $\mathrm{n}=5$ ) male rats were assessed. The percentages of strain for the four age groups when lenses were pushed with 0.05 newtons $(\mathrm{N})$ of force are shown. Data are presented as mean \pm SEM. The asterisk indicates a statistically significant difference compared to 8 -week-old ( ${ }^{*} P<0.05$, one-way analysis of variance followed by Tukey's post-hoc test).

In the present study, we measured the stiffness of the rat lens to confirm that the elasticity of the lens is decreased during aging. Further, we evaluated the effects of the application of resveratrol and two lactic acid bacteria, WB2000 and TJ515, on the stiffness of the rat lens. Both the short- and long-term administration of resveratrol and WB2000 in combination mitigated the increase in stiffness of the rat lens typically experienced during aging, whereas the administration of TJ515 alone decreased the lens stiffness with long- but not shortterm administration. These results indicate that the oral supplementation of an antioxidative diet could be a potential candidate to ameliorate the near vision impairment and presbyopia.

\section{Results}

Measurement of the lens elasticity of young and aged rats. To compare the elasticity of the lens during aging, crystalline lenses were extracted from young (8-week-old), middle-aged (24-week-old and 43-week-old) and old-aged (73-week-old) male rats and their stiffness was measured using a force-displacement measuring instrument. Previously, a coverslip lens-squeezing method and a height gauge relying on an electronic balance method were used to measure the distance of strain when pushed with a specific force ${ }^{17,31}$. In this study, we quantified the stiffness of the lens at 0.05 Newtons $(\mathrm{N})$ with the instrument (Fig. 1a-c). The group of aged rats showed decreased strain (\%, the changes of lens anteroposterior diameter) relative to that in the group of young rats, indicating that the stiffness of the lens was increased in the former (Fig. 1d; Table 1). Changes in the elasticity of lens during aging are conserved across species ranging from mice to humans ${ }^{3,5,31-33}$.

The long-term effects of resveratrol and lactic acid bacteria on the elasticity of the rat lens. To investigate the effects of resveratrol and lactic acid bacteria on rat lens elasticity, we treated male rats with resveratrol and the lactic acid bacterium WB2000 in combination or with another lactic acid bacterium, TJ515, 
a

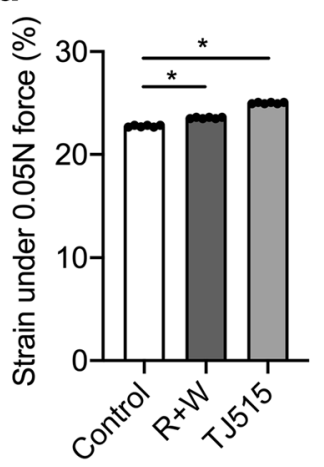

b

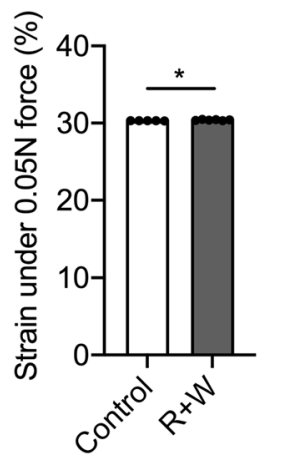

C

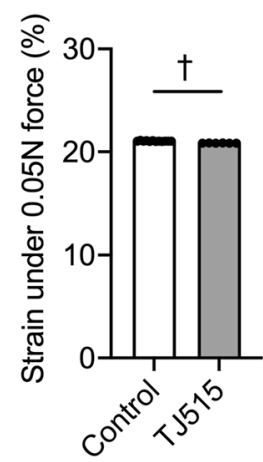

Figure 2. The effects of treatment with resveratrol and WB2000 together or TJ515 alone on rat lens elasticity. (a) Male rats (12-week-old) received the represented supplements alone or in combination by daily oral administration for 40 weeks. After the treatment period, rat lenses were extracted and their elasticity was measured ( $n=6$ in each group). The percentages of strain for the three groups (two treatment and one control) when the lenses were pushed with 0.05 Newtons $(\mathrm{N})$ of force are shown in the bar graph. (b) The effects of shortterm treatment with resveratrol and WB2000 on rat lens elasticity. Male rats (5-week-old) received resveratrol and WB2000 $(\mathrm{R}+\mathrm{W})$ in combination $(\mathrm{n}=6)$ or the vehicle (control; $\mathrm{n}=5$ ) by daily oral administration for four weeks. After the treatment period, rat lenses were extracted and their elasticity was measured. The percentages of strain when the lenses were pushed with 0.05 newtons $(N)$ of force are shown in the bar graph. (c) The effects of short-term treatment with TJ515 on rat lens elasticity. Male rats $(5$-week-old) received TJ515 $(\mathrm{n}=6)$ or vehicle (control; $n=12$ ) by daily oral administration for three weeks. After the treatment period, rat lenses were extracted and their elasticity was measured. The percentages of strain when the lenses were pushed with 0.05 newtons $(\mathrm{N})$ of force are shown in the bar graph. Data are presented as mean \pm SEM. The asterisk and dagger indicate statistically significant difference compared to each control $\left({ }^{*},{ }^{\dagger} P<0.05\right.$, one-way analysis of variance followed by Tukey's post-hoc test or Student's t-test). $\mathrm{R}+\mathrm{W}=$ Resveratrol $+\mathrm{WB} 2000$.

\begin{tabular}{|l|l|l|l|l|l|l|}
\hline \multirow{2}{*}{ Term } & \multirow{2}{*}{ Period (week) } & Supplements & Strain (\%) & $\begin{array}{l}\text { \% of strain (compared } \\
\text { to each control) }\end{array}$ & Sample number & $\begin{array}{l}\text { P value (compared } \\
\text { to each controls) }\end{array}$ \\
\hline \multirow{3}{*}{ Long-term } & \multirow{2}{*}{40} & Control & $22.75 \pm 0.08$ & 100.00 & 6 & - \\
\cline { 3 - 7 } & Resveratrol+WB2000 & $23.55 \pm 0.07$ & 103.52 & 6 & $<0.0001^{*}$ \\
\cline { 3 - 7 } & TJ515 & $25.00 \pm 0.06$ & 109.89 & 6 & $<0.0001^{*}$ \\
\hline \multirow{2}{*}{ Short-term } & \multirow{2}{*}{4} & Control & $30.32 \pm 0.01$ & 100.00 & 5 & - \\
\hline \multirow{2}{*}{ Short-term } & \multirow{2}{*}{3} & Resveratrol +WB2000 & $30.40 \pm 0.06$ & 100.26 & 6 & $<0.0001^{*}$ \\
\hline & & Control & $21.04 \pm 0.04$ & 100.00 & 12 & - \\
\cline { 3 - 7 } & TJ515 & $20.85 \pm 0.01$ & 99.10 & 6 & $<0.0001^{\dagger}$ \\
\hline
\end{tabular}

Table 2. The effects of treatment with resveratrol and WB2000 together or TJ515 alone on rat lens elasticity. As shown in Fig. 2, the percentages of strain for the three groups when lenses were pushed with 0.05 Newtons $(\mathrm{N})$ of force are shown. Data are presented as mean \pm SEM. The asterisk and dagger indicate statistically significant difference compared to each control $\left({ }^{*},{ }^{\dagger} P<0.05\right.$, one-way analysis of variance followed by Tukey's post-hoc test or Student's t-test).

alone by daily oral administration from 12 to 52 weeks of age. After 40 weeks of treatment, ocular lenses were extracted, then assessed for stiffness when pushed with $0.05 \mathrm{~N}$ of force (Fig. 2a). The combination of resveratrol and WB2000 yielded results of increased strain as compared with those of control vehicle-treated rat lenses (Fig. 2a; Table 2). Additionally, treatment with TJ515 alone for 40 weeks also increased the strain relative to that recorded in control vehicle-treated rats (Fig. 2a; Table 2). These results indicate that the elasticity of the rat lens is affected by the long-term oral administration of the combination of resveratrol and WB2000 or TJ515 alone.

The short-term effects of resveratrol and lactic acid bacteria on the elasticity of the rat lens. Considering the improvements in rat lens elasticity gained with long-term treatment with TJ515 alone or the combination of resveratrol and WB2000, we next examined whether these supplements ameliorate the stiffness of the rat lens with only short-term treatment. At this stage of the research, male rats were treated with TJ515 or the combination of resveratrol and WB2000 by daily oral administration for 4 weeks instead of 40 weeks. The distance of strain in the group of rats treated with the combination of resveratrol and WB2000 was increased, indicating that the lens elasticity was improved by oral supplementation with resveratrol and WB2000 in a short period of time (Fig. 2b; Table 2). Meanwhile, rats treated with TJ515 alone for four weeks showed decreased lens strain as compared with that measured in control vehicle-treated rats (Fig. 2c; Table 2). These 
results together indicate that the combination of resveratrol and WB2000 possesses the potential to improve rat lens elasticity both in the short and long term, while TJ515 only functions as a long-term treatment to decrease the degree of lens stiffness in rats. To gain an understanding of the effects of resveratrol and lactic acid bacteria in the context of near vision and presbyopia, a clinical study assessing resveratrol, WB2000, and TJ515 is necessary in human.

\section{Discussion}

Here, we showed that the stiffness of a crystalline lens in rats is increased with age and that daily oral administration of resveratrol and lactic acid bacteria increased the lens elasticity. In Helmholtz theory, short-range accommodation is associated with the degree of lens elasticity. Previously, we showed that lens elasticity was decreased in a smoking model in rats and pirenoxine eye drops, which have been used for the management of cataract, could improve the lens elasticity in rats ${ }^{7}$. Furthermore, the progression of near vision impairment was prevented by pirenoxine treatment in a clinical study ${ }^{7}$. Pirenoxine is known to maintain transparency in the crystalline lens by reducing the number of calcium ions, which have a crucial role in the aggregation of lens crystallin ${ }^{34,35}$. The homeostasis of lens proteins is associated with modifications including oxidation, deamidation, glycation, and the formation of disulfide bonds ${ }^{36}$. Indeed, in previous research, advanced glycation end-products mediated crosslinking and disulfide exchange among the proteins in the crystalline lens that are increased during aging ${ }^{11,37}$.

Resveratrol, contained in grapes and skin of peanuts, is used already as a dietary supplement and is known to have a beneficial effect on cancer, type 2 diabetes, and cardiovascular disease, owing to the activation of SIRT1 and its protective antioxidant properties ${ }^{38-40}$. In addition, mixed dietary supplementation containing lactoferrin, fish oil, and Enterococcus faecium WB2000 decreased the amount of ROS production from the lacrimal gland in rats ${ }^{41}$. The oxidation of cysteine thiol by ROS is known to lead to the formation of protein disulfide bonds, which is a cause of protein aggregation ${ }^{42,43}$. The antioxidant response facilitated through redox-regulated proteins and the reduced form of glutathione possesses an ability to prevent protein disulfide bonding ${ }^{44}$. Therefore, the combination of resveratrol and WB2000 may serve as an antioxidant and could improve the elasticity of the crystalline lens. Although resveratrol has the antimicrobial activity against lactic acid bacteria, adding lactic acid bacteria concurrently may support the function of gut microbiota ${ }^{45}$. Separately, we observed that treatment with Lactobacillus pentosus TJ515 isolated from fermented food in Thailand increased the elasticity of the crystalline lens in rats, so dietary supplementation with TJ515 could also have a potential implication for the near vision impairment. Some types of lactic acid bacteria, Lactobacilus species are implicated in modulating immune response on autoimmune diseases such as rheumatoid arthritis, multiple sclerosis and inflammatory bowel disease $^{46-51}$. Since aging accelerates constitutive low-grade inflammation and higher prevalence of autoimmunity even in the eye, the dietary supplement of TJ515 could also be a potential implication for the near vision ${ }^{52,53}$.

It is reported that symptoms of asthenopia including irritating and tired eyes, blurred vision, and a dry eye sensation, accompany the pre- and early stages of presbyopia ${ }^{54}$. Therefore, the treatment of presbyopia may ameliorate those symptoms of asthenopia. The pre- and early stages of presbyopia, those can be detected by the measurement of the DCNFVA (distance-corrected near functional visual acuity) begin at about 30 years old ${ }^{54,55}$. It is interesting that our short-term experiment exhibited ameliorated effects on the lens elasticity of younger rats (Fig. 2b,c). The profits of any investigation to treat the near vision impairment and presbyopia will not only improve health life expectancy but also work productivity, which will serve as checkpoints in efforts to meet sustainable development goals coordinated by the World Health Organization ${ }^{56}$.

\section{Methods}

Reagents. Resveratrol (purity $>99 \%$, observed value) extracted from Indokinoki (Pterocarpus marsupium) was purchased from Vidya Japan (Tokyo, Japan). Lactic acid bacteria, WB2000, and TJ515 were provided by Wakamoto Pharmaceutical (Tokyo, Japan).

Measurement of rat lens elasticity with a force-displacement measuring instrument with flat indentation. The elasticity of rat lenses was measured by using a force-displacement measuring instrument with flat indentation (YAWASA, Tec Gihan Co., Ltd., Tokyo, Japan). Briefly, lenses isolated from rats were immediately put on a metallic flat stage and the $12 \mathrm{~mm}$-diameter flat indenter was positioned above the lens. Then the indenter gradually applied a load on the lens along the anteroposterior axis of the lens from above until $5.0 \mathrm{~N}$ $(\mathrm{N})$ with the speed of $0.1 \mathrm{~mm} / \mathrm{s}$. The force and indentation displacement were recorded every $180^{-1} \mathrm{~s}$ to generate the force-displacement curve. We calculated the mean and SEM of the strain (\%) under $0.05 \mathrm{~N}$ and assessed the difference statistically.

Animals. We consulted the ARRIVE guideline (https://arriveguidelines.org/) to ensure proper reporting of animal experiments. The Keio University Institutional Animal Care and Use Committee approved all animal experiments (approval number: 17074), which were performed in Keio University School of Medicine, according to the Institutional Guidelines on Animal Experimentation at Keio University.

Male Wistar rats were purchased from Oriental Yeast (Tokyo, Japan). For the comparison of lens elasticity between young and aged rats, eight 8 -week-old male rats, five 24-week-old male rats, four 43-week-old male rats and five 73-week-old male rats were prepared and each groups' lenses were extracted to measure the elasticity using the YAWASA device.

For the evaluation of the effects of long-term treatment, 12 -week-old male rats were divided into six groups and orally administrated the vehicle $(n=6)$, TJ515 $(0.0070 \mathrm{mg}$ daily) $(\mathrm{n}=6)$, or a mixture of resveratrol $(0.088 \mathrm{mg}$ daily) and WB2000 (0.042 mg daily) $(n=6)$ for 40 weeks. After treatment, rat lenses were extracted to measure the elasticity using the YAWASA device. 
For the evaluation of the effects of short-term treatment with the mixture of resveratrol and WB2000, 5-weekold male rats were divided into two groups and orally administrated the vehicle $(n=5)$ or a mixture of resveratrol (0.44 mg daily) and WB2000 (0.21 mg daily) $(\mathrm{n}=6)$ for 4 weeks.

Finally, for the evaluation of the effects of short-term treatment with TJ515, 5-week-old male rats were divided into two groups and orally administrated either the vehicle $(n=12)$ or TJ515 (0.0070 mg daily) $(n=6)$ for 3 weeks. After treatment, rat lenses were extracted to measure the elasticity using the YAWASA device.

Statistical analysis. Statistical analyses were performed using GraphPad Prism (GraphPad Software, San Diego, CA, USA). Data were expressed as mean \pm standard error of the mean (SEM). The analysis of experimental data was performed using the two tailed Student's $t$-test or one-way analysis of variance, followed by Tukey's post-hoc test. A $P$ value $<0.05$ was considered to be statistically significant.

Received: 30 September 2020; Accepted: 8 January 2021

Published online: 26 January 2021

\section{References}

1. Fricke, T. R. et al. Global prevalence of presbyopia and vision impairment from uncorrected presbyopia. Ophthalmology 125, 1492-1499 (2018).

2. Reddy, P. A. et al. Effect of providing near glasses on productivity among rural Indian tea workers with presbyopia (PROSPER): a randomised trial. Lancet Glob. Health 6, e1019-e1027 (2018).

3. Heys, K. R., Cram, S. L. \& Truscott, R. J. W. Massive increase in the stiffness of the human lens nucleus with age: the basis for presbyopia?. Mol. Vis. 10, 956-963 (2004).

4. Weeber, H. A. \& van der Heijde, R. G. L. On the relationship between lens stiffness and accommodative amplitude. Exp. Eye Res. 85, 602-607 (2007).

5. Glasser, A., Campbell, C. W. \& Biometric, M. optical and physical changes in the isolated human crystalline lens with age in relation to presbyopia. Vis. Res. 39, 1991-2015 (1999).

6. Pierscionek, B. K. Age-related response of human lenses to stretching forces. Exp. Eye Res. 60, 325-332 (1995).

7. Tsuneyoshi, Y., Higuchi, A., Negishi, K. \& Tsubota, K. Suppression of presbyopia progression with pirenoxine eye drops: experiments on rats and non-blinded, randomized clinical trial of efficacy. Sci. Rep. 7, 6819 (2017).

8. Uchino, Y., Uchino, M., Dogru, M., Fukagawa, K. \& Tsubota, K. Improvement of accommodation with anti-oxidant supplementation in visual display terminal users. J. Nutr. Health Aging 16, 478-481 (2012).

9. Takahashi, Y. et al. The effect of periocular warming on accommodation. Ophthalmology 112, 1113-1118 (2005).

10. Morishita, H. \& Mizushima, N. Autophagy in the lens. Exp. Eye Res. 144, 22-28 (2016).

11. Serebryany, E., Yu, S., Trauger, S. A., Budnik, B. \& Shakhnovich, E. I. Dynamic disulfide exchange in a crystallin protein in the human eye lens promotes cataract-associated aggregation. J. Biol. Chem. 293, 17997-18009 (2018).

12. Serebryany, E. et al. An internal disulfide locks a misfolded aggregation-prone intermediate in cataract-linked mutants of human $\gamma$ D-crystallin. J. Biol. Chem. 291, 19172-19183 (2016).

13. Strenk, S. A., Semmlow, J. L., Strenk, L. M., Munoz, P. \& Gronlund-Jacob, J. Age-related changes in human ciliary muscle and lens: a magnetic resonance imaging study. Invest. Ophthalmol. Vis. Sci. 40, 8 (1999).

14. Fisher, R. F. The elastic constants of the human lens. J. Physiol. 212, 147-180 (1971).

15. Fisher, R. F. Presbyopia and the changes with age in the human crystalline lens. J. Physiol. 228, 765-779 (1973).

16. Truscott, R. J. W., Schey, K. L. \& Friedrich, M. G. Old proteins in man: a field in its infancy. Trends Biochem. Sci. 41, 654-664 (2016).

17. Garner, W. H. \& Garner, M. H. Protein disulfide levels and lens elasticity modulation: applications for presbyopia. Invest. Opthalmol. Vis. Sci. 57, 2851 (2016).

18. Karanfil, F. Ç. Update on presbyopia-correcting drops. Eur. Ophthalmic Rev. 11, 99 (2017).

19. Baur, J. A. \& Sinclair, D. A. Therapeutic potential of resveratrol: the in vivo evidence. Nat. Rev. Drug Discov. 5, 493-506 (2006).

20. Wilmarth, P. A. et al. Age-related changes in human crystallins determined from comparative analysis of post-translational modifications in young and aged lens: does deamidation contribute to crystallin insolubility?. J. Proteome Res. 5, 2554-2566 (2006).

21. Pintea, A. et al. Antioxidant effect of trans-resveratrol in cultured human retinal pigment epithelial cells. J. Ocul. Pharmacol. Ther. Off. J. Assoc. Ocul. Pharmacol. Ther. 27, 315-321 (2011).

22. Zheng, Y. et al. Resveratrol protects human lens epithelial cells against $\mathrm{H} 2 \mathrm{O} 2$-induced oxidative stress by increasing catalase, SOD-1, and HO-1 expression. Mol. Vis. 16, 1467-1474 (2010).

23. Luna, C. et al. Resveratrol prevents the expression of glaucoma markers induced by chronic oxidative stress in trabecular meshwork cells. Food Chem. Toxicol. 47, 198-204 (2009).

24. King, R. E., Kent, K. D. \& Bomser, J. A. Resveratrol reduces oxidation and proliferation of human retinal pigment epithelial cells via extracellular signal-regulated kinase inhibition. Chem. Biol. Interact. 151, 143-149 (2005).

25. Kim, Y. H., Kim, Y. S., Roh, G. S., Choi, W. S. \& Cho, G. J. Resveratrol blocks diabetes-induced early vascular lesions and vascular endothelial growth factor induction in mouse retinas. Acta Ophthalmol. (Copenh.) 90, e31-e37 (2012).

26. Nakagawa, H., Miyazaki, T. \& Department of Probiotics Immunology, Institute for Genetic Medicine, Hokkaido University, N15, W7, Kita-ku, Sapporo, Japan. Beneficial effects of antioxidative lactic acid bacteria. AIMS Microbiol. 3, 1-7 (2017).

27. Lin, X. et al. Lactic acid bacteria with antioxidant activities alleviating oxidized oil induced hepatic injury in mice. Front. Microbiol. 9, 2684 (2018).

28. Ichikawa, S., Miyake, M., Fujii, R. \& Konishi, Y. Orally administered Lactobacillus paracasei KW3110 Induces in vivo IL-12 production. Biosci. Biotechnol. Biochem. 73, 1561-1565 (2009).

29. Wakabayashi, H., Nariai, C., Takemura, F., Nakao, W. \& Fujiwara, D. Dietary supplementation with lactic acid bacteria attenuates the development of atopic dermatitis-like skin lesions in NC/Nga mice in a strain-dependent manner. Int. Arch. Allergy Immunol. 145, 141-151 (2008).

30. Morita, Y. et al. Long-term intake of Lactobacillus paracasei KW3110 prevents age-related chronic inflammation and retinal cell loss in physiologically aged mice. Aging 10, 2723-2740 (2018).

31. Cheng, C. et al. Age-related changes in eye lens biomechanics, morphology, refractive index and transparency. Aging 11, 1249712531 (2019).

32. Baradia, H., Nikahd, N. \& Glasser, A. Mouse lens stiffness measurements. Exp. Eye Res. 91, 300-307 (2010).

33. Weeber, H. A. et al. Dynamic mechanical properties of human lenses. Exp. Eye Res. 80, 425-434 (2005).

34. Liao, J.-H. et al. Ditopic complexation of selenite anions or calcium cations by pirenoxine: an implication for anti-cataractogenesis. Inorg. Chem. 50, 365-377 (2011).

35. Sharma, Y. et al. Calcium ion binding to delta- and to beta-crystallins. The presence of the 'EF-hand' motif in delta-crystallin that aids in calcium ion binding. J. Biol. Chem. 264, 12794-12799 (1989). 
36. Moreau, K. L. \& King, J. A. Protein misfolding and aggregation in cataract disease and prospects for prevention. Trends Mol. Med. 18, 273-282 (2012).

37. Nandi, S. K., Nahomi, R. B., Rankenberg, J., Glomb, M. A. \& Nagaraj, R. H. Glycation-mediated inter-protein cross-linking is promoted by chaperone-client complexes of $\alpha$-crystallin: implications for lens aging and presbyopia. J. Biol. Chem. 295, 5701-5716 (2020).

38. Jang, M. et al. Cancer chemopreventive activity of resveratrol, a natural product derived from grapes. Science 275, 218-220 (1997).

39. Baur, J. A. et al. Resveratrol improves health and survival of mice on a high-calorie diet. Nature 444, 337-342 (2006).

40. ChoSanghyun, et al. Cardiovascular protective effects and clinical applications of resveratrol. J. Med. Food. https://doi.org/10.1089/ jmf.2016.3856 (2017).

41. Kawashima, M., Nakamura, S., Izuta, Y., Inoue, S. \& Tsubota, K. Dietary supplementation with a combination of Lactoferrin, fish oil, and Enterococcus faecium WB2000 for treating dry eye: a rat model and Human Clinical Study. Ocul. Surf. 14, 255-263 (2016).

42. Hatahet, F. \& Ruddock, L. W. Protein disulfide isomerase: a critical evaluation of its function in disulfide bond formation. Antioxid. Redox Signal. 11, 2807-2850 (2009).

43. Ramkumar, S., Fan, X., Wang, B., Yang, S. \& Monnier, V. M. Reactive cysteine residues in the oxidative dimerization and Cu2+ induced aggregation of human $\gamma \mathrm{D}$-crystallin: Implications for age-related cataract. Biochim. Biophys. Acta Mol. Basis Dis. 1864, 3595-3604 (2018).

44. Chakravarthi, S., Jessop, C. E. \& Bulleid, N. J. The role of glutathione in disulphide bond formation and endoplasmic-reticulumgenerated oxidative stress. EMBO Rep. 7, 271-275 (2006).

45. García-Ruiz, A., Moreno-Arribas, M. V., Martín-Álvarez, P. J. \& Bartolomé, B. Comparative study of the inhibitory effects of wine polyphenols on the growth of enological lactic acid bacteria. Int. J. Food Microbiol. 145, 426-431 (2011).

46. Kim, J. E. et al. Lactobacillus pentosus modulates immune response by inducing IL-10 producing Tr1 cells. Immune Netw. 19, e39 (2019).

47. Liu, X. et al. Role of the gut microbiome in modulating arthritis progression in mice. Sci. Rep. 6, 30594 (2016).

48. Vaghef-Mehrabany, E. et al. Probiotic supplementation improves inflammatory status in patients with rheumatoid arthritis. Nutrition 30, 430-435 (2014).

49. Alipour, B. et al. Effects of Lactobacillus casei supplementation on disease activity and inflammatory cytokines in rheumatoid arthritis patients: a randomized double-blind clinical trial. Int. J. Rheum. Dis. 17, 519-527 (2014).

50. He, B. et al. Lactobacillus reuteri reduces the severity of experimental autoimmune encephalomyelitis in mice by modulating gut microbiota. Front. Immunol. 10, 1-12 (2019).

51. Saez-Lara, M. J., Gomez-Llorente, C., Plaza-Diaz, J. \& Gil, A. The Role of Probiotic lactic acid bacteria and bifidobacteria in the prevention and treatment of inflammatory bowel disease and other related diseases: a systematic review of randomized human clinical trials. BioMed Res. Int. vol. 2015 e505878 https://www.hindawi.com/journals/bmri/2015/505878/ (2015).

52. Xu, H., Chen, M. \& Forrester, J. V. Para-inflammation in the aging retina. Prog. Retin. Eye Res. 28, 348-368 (2009).

53. Chen, M., Luo, C., Zhao, J., Devarajan, G. \& Xu, H. Immune regulation in the aging retina. Prog. Retin. Eye Res. 69, 159-172 (2019).

54. Reindel, W., Zhang, L., Chinn, J. \& Rah, M. Evaluation of binocular function among pre- and early-presbyopes with asthenopia. Clin. Optom. 10, 1-8 (2018).

55. Katada, Y. et al. Functional visual acuity of early presbyopia. PLoS ONE 11, 1-9 (2016).

56. UN General Assembly. Transforming our world: the 2030 Agenda for Sustainable Development. Resolution adopted by the General Assembly on 25 September 2015. https://www.un.org/ga/search/view_doc.asp?symbol=A/RES/70/1\&Lang=E (2017). https://doi. org/10.1891/9780826190123.ap02.

\section{Acknowledgements}

This work was supported by Wakamoto Pharmaceutical Co., Ltd., Tokyo, Japan and Tsubota Laboratory, Inc., Tokyo, Japan. We would like to thank Pooja Gusain for proofreading the manuscript.

\section{Author contributions}

H.N., N.S., M.H., S.N., and K.T.: Design of study; H.N., S.A., and S.N.: conduct of the study; A.S.: development of force-displacement measuring instrument; H.N., N.S., and M.H.: analysis and interpretation of the data; H.N., N.S., M.H., and K.T.: preparation of the figures and manuscript. All authors have reviewed the manuscript and approved the final version.

\section{Competing interests}

K. Tsubota reports he is CEO of Tsubota Laboratory, Inc., Tokyo, Japan, a company developing products for the treatment for presbyopia. Tsubota Laboratory provides consulting services to Wakamoto Pharmaceutical Co., Ltd. and has received research funding from Wakamoto Pharmaceutical. Tsubota Laboratory, Inc. consigns the experiment to N. Sasaki, S. Nakamura, and M. Hayano. Other authors declare no conflicts of interest associated with this manuscript.

\section{Additional information}

Correspondence and requests for materials should be addressed to K.T.

Reprints and permissions information is available at www.nature.com/reprints.

Publisher's note Springer Nature remains neutral with regard to jurisdictional claims in published maps and institutional affiliations.

\footnotetext{
(c) (i) Open Access This article is licensed under a Creative Commons Attribution 4.0 International cc) License, which permits use, sharing, adaptation, distribution and reproduction in any medium or format, as long as you give appropriate credit to the original author(s) and the source, provide a link to the Creative Commons licence, and indicate if changes were made. The images or other third party material in this article are included in the article's Creative Commons licence, unless indicated otherwise in a credit line to the material. If material is not included in the article's Creative Commons licence and your intended use is not permitted by statutory regulation or exceeds the permitted use, you will need to obtain permission directly from the copyright holder. To view a copy of this licence, visit http://creativecommons.org/licenses/by/4.0/.
}

(C) The Author(s) 2021 